\title{
Capillary Surfaces in Non-Cylindrical Domains
}

\author{
G. Schindlmayr
}

\begin{abstract}
This paper is concerned with the capillary problem in a class of non-cylindrical domains in $K \subset \mathbb{R}^{n+1}$ obtained by scaling a bounded cross-section $\Omega \subset \mathbb{R}^{n}$ along the vertical axis. The capillary surfaces are described in two different ways. In the first model, they are described as the boundary of a Caccioppoli set and in a second model, after transforming $K$ to a cylinder, they are described as graphs of functions on $\Omega$. The volume of the fluid is prescribed. For both models, the energy functional is derived and declared on the appropriate function space consisting of $B V$-functions. Main results are existence and a priori bounds of minimizers, using the direct methods in the calculus of variations. For the special case of a cone over the domain $\Omega$, a criterion is given to assure that the tip is not filled with liquid. Another point of examination concerns modelling the volume restriction by means of a Lagrange multiplier.
\end{abstract}

Keywords: Equilibrium capillary surfaces, BV-functions, existence, Lagrange multiplier

AMS subject classification: 76B45, 76M30, 49Q20

\section{Introduction}

Physically it can be observed that drops of liquid are held in equilibrium in vessels that have tips at the bottom or that are unbounded with sufficently fast narrowing cross section. Examples are the cone in $\mathbb{R}^{n+1}$ over some domain $\Omega \subset \mathbb{R}^{n}$ or the narrowing tube, both shown in Figure 1.

Figure 1: Equilibrium drops in narrowing vessels

Responsible for that phenomenon are capillary forces on the free surface of the liquid and on the interface between the liquid and the boundary of the vessel. In an equilibrium configuration, the total energy of the liquid takes a (local) minimum. The

G. Schindlmayr: Kastanienweg 4, D-63303 Dreieich 
total energy is given by the sum of the surface energy, being proportional to the area of the free surface, the wetting energy, being proportional to the area of the liquid-solid interface and the potential energy. Thus, the equilibrium problem naturally leads to the formulation as a variational problem. A broad variety of mathematical methods have successfully been applied to capillary problems. A survey over many aspects of capillary surfaces and their mathematical treatment is given in [8].

In [12] the problem of capillary surfaces in a domain $K$ is treated, where $K$ is assumed to be the supergraph of a Lipschitz function $\omega$ bounded from below and satisfying $\lim _{y \rightarrow \infty} \omega(y)=\infty$. The liquid is described by a Caccioppoli set $E \subset K$, i.e. $\int_{K}\left|D \chi_{E}\right|<\infty$, with prescribed volume $|E|=V$. The advantage of such a general setting is that no assumptions about the topological type of the solution surface are needed to be made. Main results are the existence of a minimizer for the total energy and the regularity of the boundary of $E$. A regulariy result concerning the intersection curves of the vessel boundary with the liquid surface can be found in [14], formulated by means of currents from geometric measure theory (cf. [7]).

A special case extensively studied in the literature is the case where the vessel is a cylinder over some cross-section $\Omega$, such that a surface can be described by the graph of a function $u: \Omega \rightarrow \mathbb{R}$. Besides treating the Euler-Lagrange equation of the variational problem, which is an equation of prescribed mean curvature with Neumann boundary condition, advances in the existence theory have been made using the direct methods in the calculus of variations in the space $B V$ of functions of bounded variations (cf. [6, 9 11]). In [10] existence and regularity of a capillary surface in a cylinder with prescribed volume below the surface are shown.

The aim of this work is to study the capillary problem in a class of non-cylindrical domains such as those shown in Figure 1. They are obtained by scaling a cross-section $\Omega$ along the vertical coordinate $t$ via a scaling function $\phi(t)>0$. Particularly, the existence of a solution and the physical phenomenon of drops held in equilibrium, as described at the beginning of this article, are regarded. In Section 2 the liquid is modeled as a Caccioppoli set as done in [12]. For the cone $K$ over some domain $\Omega \subset \mathbb{R}^{n}$ (scaling function $\phi(t)=t$ ), a criterion is given to assure that the tip of the cone is not filled with liquid (Theorem 1). For the domain $K$, obtained by choosing $\phi(t)=\frac{1}{|t|}$ as scaling function (right-hand side of Figure 1), existence and boundedness of the solution set $E$ are shown (Theorem 2).

In Section 3 a coordinate transformation is used to transform the domain $K$ onto a cylinder, where $K$ is again obtained by scaling a cross-section $\Omega$ along the vertical axis. Then, the upper and lower surfaces of the liquid can be described by means of two functions $u$ and $v$. Motivated by [10], the volume constraint is taken into account by introducing a Lagrange multiplier. After deriving the energy functional (19), it is explained how the functional is declared for general $u, v \in B V$ (Theorem 3). For the special case of the cone (scaling function $\phi(t)=t$ ), existence and boundedness of a solution are shown and the dependence on the Lagrange multiplier $\mu$ is examined. It is proved that the volume between the surfaces monotonically converges to infinity with $\mu \rightarrow \infty$. The results are summarized in Theorem 4 .

It shall be remarked that the results from this article are part of the doctoral thesis [11], where also some more details can be found. 


\section{Model I: Caccioppoli sets}

Representing the fluid by a set $E \subset K$, the energy functional is given by

$$
\mathcal{F}(E)=\int_{K}\left|D \chi_{E}\right|+\kappa \int_{K} x_{n} \chi_{E} d x+\sigma \int_{\partial K} \chi_{E} d H_{n-1}
$$

where $\chi_{E}$ is the characteristic function of $E, \kappa>0$ is the constant of gravity and $\sigma$ is the wetting constant, which is assumed to be non-negative. The contact angle $\gamma$ is related to $\sigma$ by the identity $\sigma=-\cos \gamma$. The perimeter $\int_{K}\left|D \chi_{E}\right|$ of $E$ in $K$ is defined by

$$
\int_{K}\left|D \chi_{E}\right|=\sup \left\{\int_{K} \chi_{E} \operatorname{div} g d x \mid g \in C_{0}^{1}\left(K, \mathbb{R}^{n}\right) \text { with } \sup _{x \in K}|g(x)| \leq 1\right\} .
$$

The variational problem under consideration then is

$$
\min \mathcal{F}(E) \quad(E \in\{F \subset K:|F|=V\})
$$

Using the functional above, Giusti (cf. [12]) shows the existence and boundedness of a solution if the vessel is bounded from below and has Lipschitz-regular boundary. (Further regularity of $K$ might be needed if $\sigma<0$.) As a crucial tool to construct feasible comparison functions, Giusti used the following lemma, which will also be helpful here.

Lemma 1 (Giusti [12]). Let L be a Borel set und $D$ an open domain, $\int_{D}\left|D \chi_{L}\right|>0$. Then there exist $v_{0}>0$ and $Q_{0}>0$ depending on $D$ and $L \cap D$ such that for $|v|<v_{0}$ a set $F$ exists with $F=L$ outside of $D$ and

$$
\begin{aligned}
|F| & =|L|+v \\
\int_{D}\left|D \chi_{F}\right| & \leq \int_{D}\left|D \chi_{L}\right|+Q_{0}|v| \\
\int_{D}\left|\chi_{F}-\chi_{L}\right| d x & \leq Q_{0}|v| \int_{D}\left|\chi_{L}\right| .
\end{aligned}
$$

2.1 The Cone. Let $\Omega \subset \mathbb{R}^{n}$ be open and bounded with Lipschitz-continuous boundary $\partial \Omega$ and outer unit normal vector $\nu(x)$. Let $K \subset \mathbb{R}^{n+1}$ be the cone over $\Omega$,

$$
K=\left\{(t x, t) \mid(x, t) \in \Omega \times \mathbb{R}_{\geq 0}\right\} .
$$

This domain $K$ is a special case of the problem treated in [12]. Therefore existence and regularity of the minimizer are guaranteed. The following theorem states the condition under which the tip of the cone is not filled with liquid in equilibrium.

Theorem 1. If the domain $\Omega$ satisfies the inequality

$$
|\Omega|<\frac{\sigma}{n} \int_{\partial \Omega} \sqrt{1+(x \cdot \nu)^{2}} d H_{n-1}
$$

then for the solution $E$ of problem (3) there exists no $\varepsilon_{0}>0$ such that $\left\{x \in K \mid x_{n+1}<\right.$ $\left.\varepsilon_{0}\right\} \subset E$. 
Proof. To construct a contradiction, it is assumed that there exists an $\varepsilon_{0}$ such that $\left\{x \in K \mid x_{n+1}<\varepsilon_{0}\right\} \subset E$. Let $D \subset \subset K$ with

$$
\inf \left\{x_{n+1} \mid x \in D\right\}>\varepsilon_{0}, \quad \int_{D}\left|D \chi_{E}\right|>0, \quad \int_{\partial D}\left|D \chi_{E}\right|=0 .
$$

For notational convenience, let

$$
\begin{aligned}
& E_{\varepsilon}:=\left\{x \in E \mid x_{n+1}<\varepsilon\right\} \\
& E^{\varepsilon}:=\left\{x \in E \mid x_{n+1}>\varepsilon\right\} \\
& \Gamma_{\varepsilon}:=\left\{x \in E \mid x_{n+1}=\varepsilon\right\} .
\end{aligned}
$$

The idea of the proof is to compare the energies of $E$ and $E^{\varepsilon}$. To get a contradiction to the minimizing property of $E$, one cannot directly compare $E^{\varepsilon}$ to $E$ because $E^{\varepsilon}$ does not satisfy the volume restriction. Speaking in physical terms, the liquid being removed from the tip of the cone has to be put somewhere else (which will be inside of $D$ ) in such a way that one can still estimate the energy. To use Lemma 1 with $L=E^{\varepsilon}$ one first notices that the constants $v_{0}$ and $Q_{0}$ do not depend on $\varepsilon$ for $\varepsilon \leq \varepsilon_{0}$, since one can take the same $D$ and hence has the same sets $L \cap D$. Now $\varepsilon_{1} \leq \varepsilon_{0}$ is chosen in such a way that $\left|E_{\varepsilon}\right|<v_{0}$ for all $\varepsilon<\varepsilon_{1}$, which is always possible since clearly $\lim _{\varepsilon \rightarrow 0}\left|E_{\varepsilon}\right|=0$. By Lemma 1, for each $\varepsilon<\varepsilon_{1}$ there exists a set $F$ with $F=E^{\varepsilon}$ outside of $D$ such that (4) - (6) hold with $L=E^{\varepsilon}$ and $v=\left|E_{\varepsilon}\right|$. In particular, by (4), $|F|=\left|E_{\varepsilon}\right|+\left|E^{\varepsilon}\right|=V$, i.e. $F$ is feasible. From $(6)$ it follows

$$
\int_{K} x_{n+1} \chi_{F} d x \leq \int_{K} x_{n+1} \chi_{E^{\varepsilon}} d x+c_{1}\left|E_{\varepsilon}\right|
$$

Using $\mathcal{F}(F) \leq \mathcal{F}\left(E^{\varepsilon}\right)+c_{2}\left|E_{\varepsilon}\right|$ by (9) and (5) and comparing the energies for $E^{\varepsilon}$ and $E$, one gets

$$
\mathcal{F}(F) \leq \mathcal{F}(E)+\mathcal{H}_{n}\left(\Gamma_{\varepsilon}\right)-\sigma \int_{\partial K} \chi_{E_{\varepsilon}} d H_{n}+c_{2}\left|E_{\varepsilon}\right|
$$

where $\mathcal{H}_{n}$ denotes the $n$-dimensional Hausdorff measure. As $E_{\varepsilon}$ is just the tip of the cone by assumption, elementary integration yields

$$
\mathcal{H}_{n}\left(\Gamma_{\varepsilon}\right)=\varepsilon^{n}|\Omega| \quad \text { and } \quad \int_{\partial K} \chi_{E_{\varepsilon}} d H_{n}=\frac{\varepsilon^{n}}{n} \int_{\partial \Omega} \sqrt{1+(x \cdot \nu)^{2}} d H_{n-1}
$$

whereas $\left|E_{\varepsilon}\right|=\frac{\varepsilon^{n+1}|\Omega|}{n+1}$ is of higher order in $\varepsilon$. For $\varepsilon$ small enough, (8) leads to the contradiction $\mathcal{F}(F)<\mathcal{F}(E)$

Remark. The geometrical interpretation of the criterion given in Theorem 1 is a condition on the opening angle of the cone. The opening half-angle $\alpha$ can be defined by the identity

$$
\sin \alpha=\frac{\mathcal{H}_{n}\left(\Gamma_{\varepsilon}\right)}{\mathcal{H}_{n}\left(S_{\varepsilon}\right)}
$$


where $\mathcal{H}_{n}\left(S_{\varepsilon}\right)=\int_{\partial K} \chi_{E_{\varepsilon}} d H_{n}$ is the area of the surface $S_{\varepsilon}=\partial E_{\varepsilon} \cap \partial K$ at the tip. By (11), criterion (8) can be written in the form

$$
\frac{\mathcal{H}_{n}\left(\Gamma_{\varepsilon}\right)}{\int_{\partial K} \chi_{E_{\varepsilon}} d H_{n}}<\sigma
$$

In terms of the opening angle $\alpha$ and the contact angle $\gamma$ defined by $-\cos \gamma=\sigma$ this inequality takes the form $\sin \alpha<\sigma$ or $\alpha<\gamma-\frac{\pi}{2}$. Written in this from there are formal similarities to results in [4].

2.2 The $\frac{1}{t}$-scaled domain in $\mathbb{R}^{3}$. Let $\Omega \subset \mathbb{R}^{3}$. The domain

$$
R=\left\{\left(\frac{x}{t},-t\right) \mid(x, t) \in \Omega \times \mathbb{R}_{\geq 0}\right\}
$$

can also be parametrized by

$$
\Psi: Q=\Omega \times \mathbb{R} \rightarrow \mathbb{R}^{2} \times \mathbb{R}, \quad(x, t) \mapsto\left(e^{t} x,-e^{-t}\right) .
$$

In these local coordinates $(x, t)$, the volume element is $v_{Q}=e^{t}$. The standard coordinates in $\mathbb{R}^{3}$ will be denoted by $y$ to avoid confusion. The following version of Poincaré's inequality holds in $R$.

Lemma 2. Let $v \in B V(R)$. Then there is a constant $C=C(\Omega)$ such that

$$
\left|\int_{R} y_{3}\right| v|d y| \leq C\left(\int_{R}|D v|+\int_{\partial R}|v| d H_{2}\right) .
$$

Proof. Let $v \in B V(R)$ and $u=v \circ \Psi$ be the pull-back on $Q$. Then by Poincaré's inequality in $\Omega$

$$
\begin{aligned}
\left|\int_{R} y_{3}\right| v|d x| & =\int_{Q} e^{-t}|u| v_{Q} d x d t \\
& =\int_{\mathbb{R}} \int_{\Omega}|u(x, t)| d x d t \\
& \leq \int_{\mathbb{R}} C\left(\int_{\Omega}\left|D_{x} u\right|+\int_{\partial \Omega}|u| d H_{1}\right) d t .
\end{aligned}
$$

For smooth $v$, by coordinate transformation, one has the estimates

$$
\int_{R}|D v|=\int_{Q}\left|\left(D_{1} u, D_{2} u, x D_{1} u+y D_{2} u-D_{3} u\right)\right| d x d t \geq \int_{\mathbb{R}} \int_{\Omega}\left|D_{x} u\right| d x d t
$$

and

$$
\int_{\partial R}|v| d H_{2}=\int_{\mathbb{R}} \int_{\partial \Omega}|u| \sqrt{1+e^{4 t}(x \cdot \nu)^{2}} d H_{1} d t \geq \int_{\mathbb{R}} \int_{\partial \Omega}|u| d H_{1} d t
$$

proving the assertion for smooth $v$. For $v \in B V(R)$ the assertion follows by approximation 
To avoid the asymptotic behaviour at $y_{3}=0$, a vessel $K$ is constructed from the $\frac{1}{t}$-scaled tube $R$ by setting a truncated cone on top of $R$, such that the boundary of $K$ has infinite height. The behaviour for $t \rightarrow-\infty$ is not affected. An explicit formular for $K$ could be

$$
K:=\left\{\begin{array}{l}
\left(y, y_{3}\right) \in \mathbb{R}^{3} \mid \begin{array}{c}
y=x /\left|y_{3}-1\right| \text { for } y_{3}<0 \\
y=x\left(y_{3}+1\right) \text { for } y_{3}>0
\end{array}
\end{array}(x \in \Omega)\right\}
$$

What remains to be examined is the question whether a drop can be in equilibrium without "falling down" the vessel. An answer is given in the theorem below.

Theorem 2. Let $\mathcal{F}$ be the functional defined in $(1), 0<\sigma \leq 1$. Let $\kappa$ be sufficently small. Then there exists a set $E \subset K$ minimizing $\mathcal{F}$ among all sets $F \subset K$ with $|F|=V$ und $\inf \left\{y_{3} \mid y \in F\right\}>-\infty$.

Proof. The first step is to show that the energy functional (1) is bounded from below. For $E \subset K, E_{0}=E \cap\left\{y_{3}<0\right\}$, one can apply Lemma 2 to get

$$
\begin{aligned}
\kappa \int_{K} y_{3} \chi_{E} d y & \geq \kappa \int_{K} y_{3} \chi_{E_{0}} d y \\
& \geq-\kappa C\left(\int_{K}\left|D \chi_{E_{0}}\right|+\int_{\partial K} \chi_{E_{0}} d H_{2}\right) \\
& \geq-\left(\int_{K}\left|D \chi_{E}\right|+\sigma \int_{\partial K} \chi_{E} d H_{2}+|\Omega|\right)
\end{aligned}
$$

for $\kappa \leq \frac{\sigma}{C}$. The boundedness of (2) now follows immediately.

The next step is to find a minimizer among all sets $F \subset K^{-M}$, where $K^{-M}=\{y \in$ $\left.K \mid y_{3}>-M\right\}$ is the vessel with an artificial obstacle at level $-M$. The vessel $K^{-M}$ fits into the framework of [12], thus the existence (and $C^{1, \alpha}$-regularity) is ensured.

The last step is to show that the obstacle is not touched, such that for $M \geq M_{0}$ one has the same minimizer. This follows from Lemma 3, where an a priori lower bound for the minimizer is shown, that is independent of $M$

Lemma 3. Let $\mathcal{F}$ be the functional defined in (1), $0<\sigma \leq 1$ and $\kappa$ be sufficently small. Let $E$ minimize $\mathcal{F}$ among all $F \subset K^{-M},|F|=V$ where $K^{-M}=\left\{y \in K \mid y_{3}>\right.$ $-M\}$. Then there exists a bound $M_{0}$ independent of $M$ such that $\inf \left\{y_{3} \mid y \in F\right\}>-M_{0}$.

Proof. The idea of the proof follows [12] where upper bounds are shown. Let

$$
\begin{aligned}
& E_{T}:=\left\{y \in E \mid y_{3}<T\right\} \\
& E^{T}:=\left\{y \in E \mid y_{3}>T\right\} \\
& \Gamma_{T}:=\left\{y \in E \mid y_{3}=T\right\} .
\end{aligned}
$$

Let $D \subset \subset K$ open with $\int_{D}\left|D \chi_{E}\right|>0$ and $\int_{\partial D}\left|D \chi_{E}\right|=0$. For $T<T_{0} \leq \inf \left\{y_{3} \mid y \in D\right\}$ one has $\left|E_{T}\right|<v_{0}$ and by Lemma 1 there is a set $F$ with $F=E_{T}$ outside of $D$ such that (4) - (6) hold with $v=\left|E_{T}\right|$. In particular, $|F|=V$, i.e. $F$ is feasible, and

$$
\int_{K} y_{3} \chi_{F} d x \leq \int_{K} y_{3} \chi_{E^{T}} d x+c_{1}\left|E_{T}\right|
$$


By comparing $F$ and $E$, one gets

$$
\begin{aligned}
\mathcal{F}(F) \leq & \mathcal{F}\left(E^{T}\right)+c_{2}\left|E_{T}\right| \\
\leq & \mathcal{F}(E)+2 \mathcal{H}_{2}\left(\Gamma_{T}\right)-\int_{K}\left|D \chi_{E_{T}}\right| \\
& -\sigma \int_{\partial K} \chi_{E_{T}} d H_{2}-\kappa \int_{K} y_{3} \chi_{E_{T}} d x+c_{2}\left|E_{T}\right| .
\end{aligned}
$$

Using Lemma 2 and subsequently the isoperimetric inequality gives

$$
\begin{aligned}
\mathcal{F}(F) & \leq \mathcal{F}(E)+2 \mathcal{H}_{2}\left(\Gamma_{T}\right)-(1-a)\left(\int_{K}\left|D \chi_{E_{T}}\right|+\sigma \int_{\partial K} \chi_{E_{T}} d H_{2}\right)+c_{2}\left|E_{T}\right| \\
& \leq \mathcal{F}(E)+2 \mathcal{H}_{2}\left(\Gamma_{T}\right)-(1-a) \sigma \int_{\mathbb{R}^{3}}\left|D \chi_{E_{T}}\right|+c_{2}\left|E_{T}\right| \\
& \leq \mathcal{F}(E)+2 \mathcal{H}_{2}\left(\Gamma_{T}\right)-c_{3}\left|E_{T}\right|^{1-\frac{1}{n}}+c_{2}\left|E_{T}\right| \\
& \leq \mathcal{F}(E)+2 \mathcal{H}_{2}\left(\Gamma_{T}\right)-c_{4}\left|E_{T}\right|^{1-\frac{1}{n}}
\end{aligned}
$$

for $T<T_{0}$ where in the last step $\left|E_{T}\right| \rightarrow 0$ for $T \rightarrow-\infty$ was used. Since $E$ is a minimizer,

$$
2 \mathcal{H}_{2}\left(\Gamma_{T}\right)-c_{4}\left|E_{T}\right|^{1-\frac{1}{n}} \geq 0
$$

The remaining steps are similar to [12]: From (14) it follows that

$$
\frac{d}{d T}\left|E_{T}\right|=\mathcal{H}_{2}\left(\Gamma_{T}\right) \geq \frac{c_{4}}{2}\left|E_{T}\right|^{1-\frac{1}{n}}
$$

Assuming $E_{T}>0$ for $T<T_{0}$, integrating the last inequality leads to

$$
\left|E_{T_{0}}\right|^{\frac{1}{n}}-\left|E_{T}\right|^{\frac{1}{n}} \geq \frac{c_{4}}{2 n}\left(T_{0}-T\right)
$$

and thus $T \geq T_{0}-\frac{2 n}{c_{4}}\left|E_{T_{0}}\right|^{\frac{1}{n}}$

\section{Model II: non-parametric surfaces}

3.1 General scaled vessels. Let the vessel $K$ be constructed by scaling a cross-section $\Omega \subset \mathbb{R}^{n}$ along the vertical axis,

$$
K=\{(\phi(t) x, t) \mid(x, t) \in \Omega \times \mathbb{R}\} .
$$

The scaling function $\phi$ is assumed to be smooth. To represent surfaces in $K$ by graphs of functions, one can use local coordinates, that transform $K$ onto the cylinder $\Omega \times \mathbb{R}$. Such local coordinates are given by the map

$$
\Psi: Q=\Omega \times \mathbb{R} \rightarrow \mathbb{R}^{n+1}, \quad(x, t) \mapsto(\phi(t) x, t) .
$$


For the moment, let $\partial \Omega$ be smooth. The induced metric tensors $g^{K}$ on $K$ and $g^{\partial K}$ on $\partial K$ are

$$
\begin{aligned}
g^{K} & =\left(\begin{array}{cc}
\phi^{2} \mathrm{I}_{n} & \phi \phi^{\prime} x \\
\phi \phi^{\prime} x^{T} & 1+\left(\phi^{\prime}\right)^{2}|x|^{2}
\end{array}\right) \\
g^{\partial K} & =\left(\begin{array}{cc}
\phi^{2} \mathrm{I}_{n-1} & \phi \phi^{\prime} P x \\
\phi \phi^{\prime}(P x)^{T} & 1+\left(\phi^{\prime}\right)^{2}|x|^{2}
\end{array}\right)
\end{aligned}
$$

where $P$ is the orthogonal projection onto $T(\partial \Omega)$. The induced metric tensor on a surface $\mathcal{S}(u): t=u(x)$ is

$$
g^{\mathcal{S}}=\phi^{2} \mathrm{I}_{n}+\phi \phi^{\prime}(x \otimes D u+D u \otimes x)+\left(1+|x|^{2}\right) D u \otimes D u
$$

The corresponding volume elements $\sqrt{\operatorname{det} g}$ are $v_{K}=\phi^{n}$,

$$
\begin{aligned}
v_{\partial K} & =\phi^{n-1} \sqrt{1+\left(\phi^{\prime}\right)^{2}(x \cdot \nu)^{2}} \\
v_{\mathcal{S}} & =\left(\phi^{2(n-1)}(u)|D u|^{2}+\left(\operatorname{div}\left(\phi^{n}(u) x\right)\right)^{2}\right)^{\frac{1}{2}} .
\end{aligned}
$$

The energy functional for a liquid between the surfaces $\mathcal{S}\left(u_{1}\right)$ and $\mathcal{S}\left(u_{2}\right)$, where $u_{1}$ and $u_{2}$ are smooth functions, can be written as

$$
\begin{aligned}
J\left(u_{1}, u_{2}\right)= & \int_{\Omega} v_{\mathcal{S}\left(u_{1}\right)}+v_{\mathcal{S}\left(u_{2}\right)} d x \\
& +\kappa \int_{\Omega} \int_{u_{1}}^{u_{2}} t v_{K} d t d x+\sigma \int_{\partial \Omega} \int_{u_{1}}^{u_{2}} v_{\partial K} d t d H_{n-1} .
\end{aligned}
$$

After another transformation $v=\Phi(u)$ with $\Phi^{\prime}=\phi^{n-1}>0$ and $\Phi(0)=0$ and after introducing a Lagrange multiplier $\mu$ for the volume restriction, the final version of the energy functional is (writing $u$ and $v$ instead of $v_{1}$ and $v_{2}$ )

$$
\begin{aligned}
\mathcal{F}_{\mu}(u, v)= & \int_{\Omega}\left(|D u|^{2}+(\operatorname{div}(\psi(u) x))^{2}\right)^{\frac{1}{2}}+\int_{\Omega}\left(|D v|^{2}+(\operatorname{div}(\psi(v) x))^{2}\right)^{\frac{1}{2}} \\
& +\int_{\Omega} \int_{u(x)}^{v(x)} H_{\mu}(t) d t d x+\sigma \int_{\partial \Omega} \int_{u(x)}^{v(x)} \sqrt{1+\left(\psi^{\prime}(t)\right)^{2}(x \cdot \nu)^{2}} d t d H_{n-1} .
\end{aligned}
$$

Here, $\psi=\phi^{n} \circ \Phi^{-1} / n, H_{\mu}=\kappa \zeta \Phi^{-1}-\mu \zeta$ and $\zeta=\phi \circ \Phi^{-1}$.

In analogy to the capillary problem in the cylinder (cf. $[6,9-11]$ ), a minimum is sought in the class $B V$ of functions of bounded variation. This is due to the linear growth of $\mathcal{F}$ in the gradients of $u$ and $v$. Therefore the functional $\mathcal{F}$ has to be defined for general $B V$-functions. This can be done via a measure-theoretical method developed in [5] (cf. also $[1,2])$.

The following theorem holds.

Theorem 3. Let $\Omega \subset \mathbb{R}^{n}$ be bounded and $f \in C\left(\bar{\Omega} \times \mathbb{R} \times \mathbb{R}^{n}\right)$, such that

$$
m|p|-a \leq f(x, s, p) \leq M|p|+A \quad\left((x, s, p) \in \Omega \times \mathbb{R} \times \mathbb{R}^{n}\right)
$$


with constants $m>0$ and $a, A, M \geq 0$. For every $(x, s) \in \Omega \times \mathbb{R}$ let the map $p \mapsto$ $f(x, s, p)$ be convex on $\mathbb{R}^{n}$. Then the functional $u \mapsto \int_{\Omega} f(x, u, D u) d x$ can be extended to the functional $u \mapsto I(u) \equiv \int_{\Omega} f(x, u, D u)$ on $B V_{l o c}(\Omega)$, such that:

1. $I(u)$ is lower semi-continuous on $B V_{l o c}(\Omega)$ with respect to $L_{l o c}^{1}(\Omega)$-convergence.

2. For $u \in B V(\Omega) \cap L^{\infty}(\Omega)$ there exists a sequence $\left\{u_{k}\right\}$ of functions $u_{k} \in C^{1}(\Omega)$ such that $u_{k} \rightarrow u$ in $L^{1}(\Omega)$ and $\int_{\Omega} f\left(x, u_{k}, D u_{k}\right) \rightarrow \int_{\Omega} f(x, u, D u)$ for $j \rightarrow \infty$.

The construction $\int_{\Omega} f(x, u, D u)$ shall shortly be reviewed. For a Radon measure $\lambda$, let $\lambda=\lambda^{a}+\lambda^{s}$ be the Lebesgue decomposition in an absolutely continuous and a singular part with respect to the Lebesgue measure $\mathcal{L}^{n}$. The $\mathcal{L}^{n}$-density of $E$ in $x$ is defined as

$$
\lim _{\rho \rightarrow 0^{+}} \frac{\left|E \cap B_{\rho}(x)\right|}{\left|B_{\rho}(x)\right|} \quad \text { where } B_{\rho}(x)=\left\{y \in \mathbb{R}^{n}|| y-x \mid<\rho\right\} .
$$

Let

$$
f^{0}: \Omega \times R \times \mathbb{R}^{n} \rightarrow \mathbb{R}_{+}, \quad f^{0}(x, s, p)=\lim _{t \rightarrow 0^{+}} f(x, s, p / t) t
$$

and

$$
\tilde{f}: \Omega \times R \times \mathbb{R}^{n} \times \mathbb{R}_{-} \rightarrow \mathbb{R}^{+}, \quad \tilde{f}(x, s, p, t)=\left\{\begin{array}{ll}
-f(x, s,-p / t) t & \text { if } t<0 \\
f^{0}(x, s, p) & \text { if } t=0
\end{array} .\right.
$$

Then $(p, t) \mapsto \tilde{f}(x, s, p, t)$ is convex and homogeneous of degree one on $\mathbb{R}^{n} \times \mathbb{R}_{-}$. The following definitions are still needed: Let $M(u) \subset \Omega$ be a Borel set with

$$
\mathcal{L}^{n}(M(u))=|D u|^{s}(\Omega-M(u))=0 .
$$

Furthermore, let

$$
\begin{aligned}
u_{-} & =\sup \left\{t \mid\{y \mid u(y)<t\} \text { has } \mathcal{L}^{n}-\text { density } 0 \text { in } x\right\} \\
u_{+} & =\inf \left\{t \mid\{y \mid u(y)>t\} \text { has } \mathcal{L}^{n}-\text { density } 0 \text { in } x\right\} \\
N(u) & =\left\{x \in \Omega \mid u_{-}(x)<u_{+}(x)\right\} \\
S(u) & =\left\{(x, s) \in \Omega \times \mathbb{R} \mid s<u_{+}(x)\right\} .
\end{aligned}
$$

Then $\int_{\Omega} f(x, u, D u)$ is defined by the identity

$$
\begin{aligned}
\int_{\Omega} f(x, u, D u)= & \int_{\Omega} f\left(x, u(x),(D u)^{a}(x)\right) d x \\
& +\int_{M(u)-N(u)} f^{0}\left(x, u^{+}(x), \frac{d D u}{d|D u|}(x)\right) d|D u| \\
& +\int_{N(u)} \int_{u^{-}(x)}^{u^{+}(x)} f^{0}\left(x, s, \frac{d D u}{d|D u|}(x)\right) d \mathcal{H}^{n-1} .
\end{aligned}
$$

Evaluating the divergence term in the integrand $\left(|D u|^{2}+(\operatorname{div}(\psi(u) x))^{2}\right)^{\frac{1}{2}}$, Theorem 2 can be applied to the functional $\mathcal{F}$ in (19) setting

$$
f(x, s, p)=\left(|p|^{2}+\left(n \psi(s)+\psi^{\prime}(s) p \cdot x\right)^{2}\right)^{\frac{1}{2}} .
$$


The convexity of $f$ can be checked via the Hessian matrix. For details of the relaxation procedure cf. [13].

In the following subsection, the special case where $K$ is a cone is treated in greater detail. Applying the non-parametric methods to the case where $K$ is $\frac{1}{t}$-scaled, one gets similar results corresponding to those from Subsection 2.2.

3.2 Non-parametric surfaces in the cone. The cone is a special case of the vessels considered in (15) with scaling function $\phi(t)=t$. The total energy functional (19) becomes

$$
\begin{aligned}
\mathcal{F}_{\mu}(u, v):= & \int_{\Omega}\left(|D u|^{2}+(\operatorname{div}(u x))^{2}\right)^{\frac{1}{2}}+\int_{\Omega}\left(|D v|^{2}+(\operatorname{div}(v x))^{2}\right)^{\frac{1}{2}} \\
& +\int_{\Omega} \kappa\left(v^{\frac{n+2}{n}}-u^{\frac{n+2}{n}}\right)-\mu\left(v^{\frac{n+1}{n}}-u^{\frac{n+1}{n}}\right) d x \\
& +\sigma \int_{\partial \Omega}(v-u) \sqrt{1+(x \cdot \nu)^{2}} d H_{n-1} .
\end{aligned}
$$

Remark. As an alternative to the general theory behind Theorem 3, the surface area functional for the cone can be defined by

$$
\begin{aligned}
& \int_{\Omega}\left(|D u|^{2}+(\operatorname{div}(u x))^{2}\right)^{\frac{1}{2}} \\
& \quad=\sup \left\{\int_{\Omega} u \operatorname{div} g+u x \cdot D g_{n+1} d x \mid \begin{array}{l}
\mathbf{g}=\left(g, g_{n+1}\right) \in C_{0}^{1}\left(\Omega, \mathbb{R}^{n+1}\right) \\
\sup _{x \in \Omega}|\mathbf{g}(x)| \leq 1
\end{array}\right\} .
\end{aligned}
$$

A similar technique was used in [3]. Property 1 (lower semi-continuity) from Theorem 3 is easily checked, whereas property 2 (smooth approximation) is more complicated and proved by using mollifiers (cf. [13]).

The aim of this section is to prove the following theorem.

Theorem 4 (Summary of Theorems 5 - 9). Let $\partial \Omega$ be of class $C^{2}$ and $0<\sigma \leq$ $1-a(a>0)$. Then for $\mu>0$ the problem $\mathcal{F}_{\mu}(u, v) \rightarrow \min$ has a solution in

$$
K_{\delta}=\left\{(u, v) \in(B V(\Omega))^{2} \cap\left(L^{\infty}(\Omega)\right)^{2} \mid 0 \leq u \leq \delta \leq v\right\} .
$$

The solution functions $u_{\mu}$ and $v_{\mu}$ satisfy the estimate

$$
0 \leq u_{\mu} \leq v_{\mu} \leq k_{\mu} \equiv\left(\frac{(n+1) \mu}{(n+2) \kappa}\right)^{n} .
$$

The sequences $\left\{u_{\mu}\right\}$ and $\left\{v_{\mu}\right\}$ are non-increasing and non-decreasing in $\mu$, respectively. For $\mu \rightarrow \infty$ one has

$$
\begin{aligned}
& \lim _{\mu \rightarrow \infty} \inf _{\Omega} v_{\mu}=\infty \\
& \lim _{\mu \rightarrow \infty} \sup _{\Omega} u_{\mu}=0 .
\end{aligned}
$$

If $|\Omega|<\frac{\sigma}{n} \int_{\partial \Omega} \sqrt{1+(x \cdot \nu)^{2}} d H_{n-1}$, then $u$ is not identical to zero on $\Omega$.

The monotony in $\mu$ and the limits (24) - (25) describe how the solution depends on the Lagrange multiplier. In particular, the volume of the liquid, $V=\int v_{\mu}^{\frac{n+1}{n}}-u_{\mu^{\frac{n+1}{n}}} d x \rightarrow$ $\infty$ as $\mu \rightarrow \infty$. For clearness of the proof, the theorem is split into the sequence of Theorems $5-9$. 
Theorem 5 (Existence). Let $\partial \Omega$ be of Lipschitz type and $0 \leq \sigma \leq 1$. Then the problem

$$
\mathcal{F}_{\mu}(u, v) \rightarrow \min \quad \text { in } K_{\delta}
$$

has a solution $(u, v)$ satisfying $v \leq k_{\mu} \equiv\left(\frac{(n+1) \mu}{(n+2) \kappa}\right)^{n}$.

Proof. As a first step, the a priori estimate for $v$ shall be derived. Let $v_{0}=$ $\min \left(v, k_{\mu}\right)$ and $A=\left\{x \in \Omega \mid v(x)>k_{\mu}\right\}$. Assume $|A|>0$. Clearly,

$$
\begin{gathered}
\int_{\Omega}\left(\left|D v_{0}\right|^{2}+\operatorname{div}\left(v_{0} x\right)^{2}\right)^{\frac{1}{2}} \leq \int_{\Omega}\left(|D v|^{2}+\operatorname{div}(v x)^{2}\right)^{\frac{1}{2}} \\
\sigma \int_{\partial \Omega}\left(v_{0}-u\right) \sqrt{1+(x \cdot \nu)^{2}} d H_{n-1} \leq \sigma \int_{\partial \Omega}(v-u) \sqrt{1+(x \cdot \nu)^{2}} d H_{n-1} .
\end{gathered}
$$

The function $f_{\mu}(x)=\kappa x^{\frac{n+2}{n}}-\mu x^{\frac{n+1}{n}}$ takes its strict minimum in $k_{\mu}$, so for the potential energy term,

$$
\int_{\Omega} f_{\mu}\left(v_{0}\right)-f_{\mu}(u) d x<\int_{\Omega} f_{\mu}\left(v_{0}\right)-f_{\mu}(u) d x
$$

by the assumption. Those three inequalities give $\mathcal{F}_{\mu}\left(u, v_{0}\right)<\mathcal{F}_{\mu}(u, v)$, which is a contradiction to the minimizing property of $(u, v)$. Therefore, $v \leq k_{\mu}$. Now the existence can be proved. By the a priori estimate, the solution is sought in $\tilde{K}_{\delta}=\left\{u \in K_{\delta} \mid u \leq k_{\mu}\right\}$. Since $H_{\mu}(t) \rightarrow \infty$ for $t \rightarrow \infty$, the functional $\mathcal{F}_{\mu}$ is bounded from below on $K_{\delta}$. Any minimizing sequence $\left(u_{k}, v_{k}\right) \quad(k \in \mathbb{N})$ is bounded in $(B V(\Omega))^{2}$. By compactness of the embedding $B V(\Omega) \hookrightarrow L^{1}(\Omega)$, there is a subsequence that converges in $\left(L^{1}(\Omega)\right)^{2}$ to a $(u, v) \in\left(L^{1}(\Omega)\right)^{2}$. Lower semicontinuity of the functional implies that $(u, v)$ is a minimizer. One easily checks that $(u, v) \in \tilde{K}_{\delta}$

Theorem 6 (Monotonicity in $\mu$ ). Let $\partial \Omega$ be of Lipschitz type and $0 \leq \sigma \leq 1$. For $i \in\{1,2\}$ and $\mu_{1}<\mu_{2}$, let $\left(u_{i}, v_{i}\right)$ be the solution of

$$
\mathcal{F}_{\mu_{i}}(u, v) \rightarrow \min \quad \text { in } K_{\delta} .
$$

Then $v_{1} \leq v_{2}$ and $u_{1} \geq u_{2}$ a.e.

Proof. For notational convenience, let

$$
\begin{aligned}
v_{0} & =\max \left(v_{1}, v_{2}\right) \\
u_{0} & =\min \left(u_{1}, u_{2}\right) \\
f_{\mu}(x) & =\kappa x^{\frac{n+2}{n}}-\mu x^{\frac{n+1}{n}} \\
I_{\mu}(u) & =\int_{\Omega}\left(|D u|^{2}+(\operatorname{div}(u x))^{2}\right)^{\frac{1}{2}}-\int_{\Omega} f_{\mu}(u) d x-\sigma \int_{\partial \Omega} u \sqrt{1+(x \cdot \nu)^{2}} d H_{n-1} \\
J_{\mu}(v) & =\int_{\Omega}\left(|D v|^{2}+(\operatorname{div}(v x))^{2}\right)^{\frac{1}{2}}+\int_{\Omega} f_{\mu}(v) d x+\sigma \int_{\partial \Omega} v \sqrt{1+(x \cdot \nu)^{2}} d H_{n-1} .
\end{aligned}
$$

Here, only $v_{1} \leq v_{2}$ will be shown. Let $A=\left\{x \in \Omega \mid v_{1}>v_{2}\right\}$ and $w_{0}=\min \left(v_{1}, v_{2}\right)$. Since $\left(u_{1}, v_{1}\right)$ minimizes $\mathcal{F}_{\mu_{1}}$,

$$
I_{\mu_{1}}\left(u_{1}\right)+J_{\mu_{1}}\left(v_{1}\right)=\mathcal{F}_{\mu_{1}}\left(u_{1}, v_{1}\right) \leq \mathcal{F}_{\mu_{1}}\left(u_{1}, w_{0}\right)=I_{\mu_{1}}\left(u_{1}\right)+J_{\mu_{1}}\left(w_{0}\right),
$$


hence $J_{\mu_{1}}\left(v_{1}\right) \leq J_{\mu_{1}}\left(w_{0}\right)$. Similarly, $J_{\mu_{2}}\left(v_{2}\right) \leq J_{\mu_{2}}\left(v_{0}\right)$ holds. Together, this gives

$$
J_{\mu_{1}}\left(v_{1}\right)+J_{\mu_{2}}\left(v_{2}\right) \leq J_{\mu_{1}}\left(w_{0}\right)+J_{\mu_{2}}\left(v_{0}\right) .
$$

Using the definitions of $v_{0}$ and $w_{0}$ and using $v_{0}+w_{0}=v_{1}+v_{2}$, the identity

$$
\begin{aligned}
J_{\mu_{1}}\left(w_{0}\right)+J_{\mu_{2}}\left(v_{0}\right)= & J_{\mu_{1}}\left(v_{1}\right)+J_{\mu_{2}}\left(v_{2}\right) \\
& +\int_{A} f_{\mu_{1}}\left(v_{2}\right)+f_{\mu_{2}}\left(v_{1}\right)-f_{\mu_{1}}\left(v_{1}\right)-f_{\mu_{2}}\left(v_{2}\right) d x .
\end{aligned}
$$

can easily be derived. Simplifying the last term by using the definition of $f_{\mu}$ and by using $v_{1}>v_{2}$ on $A$, this leads to

$$
f_{\mu_{1}}\left(v_{2}\right)+f_{\mu_{2}}\left(v_{1}\right)-f_{\mu_{1}}\left(v_{1}\right)-f_{\mu_{2}}\left(v_{2}\right)=\left(\mu_{1}-\mu_{2}\right)\left(v_{1}^{\frac{n+1}{n}}-v_{2}^{\frac{n+1}{n}}\right) \leq 0
$$

on $A$. Equality only holds if $|A|=0$. Suppose, $|A|>0$. Then, by the last estimate,

$$
J_{\mu_{1}}\left(w_{0}\right)+J_{\mu_{2}}\left(v_{0}\right)<J_{\mu_{1}}\left(v_{1}\right)+J_{\mu_{2}}\left(v_{2}\right)
$$

in contradiction to (28). Therefore, $|A|=0$ or, equivalently, $v_{1} \leq v_{2}$ a.e.

For proving the following theorems, some lemmata are needed. The first one concerns the domain $\Omega$.

Lemma 4. Let $\partial \Omega$ be of class $C^{2}$. Then there exists a constant $C_{\varepsilon}$ such that for $v, u \in B V(\Omega)$ with $v \geq u \geq \varepsilon$ and $A=\{x \in \Omega \mid u(x)<v(x)\}$

$$
\begin{aligned}
& \int_{\partial \Omega}(v-u) \sqrt{1+(x \cdot \nu)^{2}} d H_{n-1} \\
& \quad \leq \int_{A}\left(|D v|^{2}+(\operatorname{div}(v x))^{2}\right)^{\frac{1}{2}}+\int_{A}\left(|D u|^{2}+(\operatorname{div}(u x))^{2}\right)^{\frac{1}{2}}+C_{\varepsilon} \int_{A} v^{\frac{n+1}{n}}-u^{\frac{n+1}{n}} d x .
\end{aligned}
$$

It shall be remarked that the tip of the cone is taken out of consideration by introducing the level $\varepsilon$. Then this lemma is the non-parametric version of the following lemma stated in [12].

Lemma 5. Let $K \subset \mathbb{R}^{n+1}$ be the supergraph of some $C^{2}$-function $\omega$ with bounded mean curvature. Then there exists a constant $C>0$ such that for all $A \subset K$

$$
\int_{\partial K} \chi_{A} d H_{n-1} \leq \int_{K}\left|D \chi_{A}\right|+C|A| .
$$

Another lemma concerning level-sets will be used later to prove a priori bounds for the solutions.

Lemma 6. Let $\Omega \subset \mathbb{R}^{n}$ be bounded, $f \in L^{1}(\Omega)$ and $A(\lambda)=\left\{x \in \mathbb{R}^{n} \mid f(x)<\lambda\right\}$. Then the non-decreasing function

$$
y: \mathbb{R} \rightarrow \mathbb{R}, \quad \lambda \mapsto \int_{A(\lambda)} \lambda-f(x) d x
$$


is continuous and differentiable from the left with $y_{-}^{\prime}(\lambda)=|A(\lambda)|$.

Proof. By definition of $y(\lambda)$, one has for $h>0$

$$
\begin{aligned}
& y(\lambda)-y(\lambda-h) \\
& =\int_{A(\lambda)} \lambda-f(x) d x-\int_{A(\lambda-h)} \lambda-h-f(x) d x \\
& =\int_{A(\lambda-h)} \lambda-f(x) d x+\int_{A(\lambda)-A(\lambda-h)} \lambda-f(x) d x-\int_{A(\lambda-h)} \lambda-h-f(x) d x \\
& =\int_{A(\lambda-h)} h d x+\int_{A(\lambda)-A(\lambda-h)} \lambda-f(x) d x .
\end{aligned}
$$

Therefore, since $\lambda-h \leq f(x)<\lambda$ in $A(\lambda)-A(\lambda-h)$,

$$
|y(\lambda)-y(\lambda-h)| \leq h|A(\lambda-h)|+h|A(\lambda)-A(\lambda-h)| .
$$

This gives the continuity of $y$ in $\lambda$. It remains to show that $y_{-}^{\prime}(\lambda)=|A(\lambda)|$, which is equivalent to

$$
\lim _{h \rightarrow 0^{+}}\left|\frac{y(\lambda)-y(\lambda-h)}{h}-\right| A(\lambda) \mid=0 .
$$

By the above calculation, one has the estimate

$$
\begin{aligned}
\left|\frac{y(\lambda)-y(\lambda-h)}{h}-\right| A(\lambda)|| & \leq|| A(\lambda-h)|+| A(\lambda)-A(\lambda-h)|-A(\lambda)| \\
& \leq 2|A(\lambda)-A(\lambda-h)| .
\end{aligned}
$$

The right-hand side converges to zero for $h \rightarrow 0^{+}$if

$$
\chi_{A(\lambda-h)} \rightarrow \chi_{A(\lambda)} \quad \text { for } h \rightarrow 0^{+} \text {in } L^{1}(\Omega) .
$$

Relation (30) can be shown by Lebesgue's convergence theorem. Since the characteristic function is dominated by 1 , it suffices to show the pointwise convergence. Let $x \in \Omega$ be given. If $\chi_{A(\lambda)}(x)=0$, then this implies $\chi_{A(\lambda-h)}(x)=0$ for all $h>0$ and the convergence is trivial. The other case is $\chi_{A(\lambda)}(x)=1$. If one had $\chi_{A(\lambda-h)}(x)=0$ for all $h>0$, then this would lead to the contradiction $\lambda-h \leq f(x)<\lambda$ for all $h>0$. Therefore, $\chi_{A\left(\lambda-h_{0}\right)}(x)=1$ for a $h_{0}>0$. But then $\chi_{A(\lambda-h)}(x)=1$ holds for all $0<h \leq h_{0}$, again showing the convergence for $h \rightarrow 0^{+}$

As a last tool, the following version of a comparison theorem for ordinary differential equations, similar to Gronwall's lemma is stated.

Lemma 7. Let $y:[a, b] \rightarrow \mathbb{R}$ be non-decreasing and continuous, $f$ be non-decreasing and Lipschitz continuous and $g \geq 0$ be continuous, such that the inequality $y^{\prime}(x) \geq$ $f(y(x)) g(x)$ holds a.e. Let $\psi$ be the (unique) solution of the initial value problem

$$
\left.\begin{array}{rl}
\psi^{\prime}(x) & =f(\psi(x)) g(x) \\
\psi(a) & =y(a)
\end{array}\right\} .
$$


Then $y(x) \geq \psi(x)$ for all $x \in[a, b]$.

Theorem 7. Let $\lambda_{1}>0$ be given. Then under the assumptions of Theorem 4 there exists a $\mu_{0}$ such that

$$
v_{\mu} \geq \lambda_{1}
$$

a.e. for $\mu \geq \mu_{0}$.

Proof. Let $\lambda_{2}>\lambda_{1}$ and $\lambda_{2} \geq \lambda \geq \lambda_{1}$. Define

$$
\begin{aligned}
A(\lambda) & =\left\{x \in \Omega \mid v_{\mu}(x)<\lambda\right\} \\
v_{\mu, \lambda} & =\max \left(v_{\mu}, \lambda\right) \\
w_{\mu, \lambda} & =v_{\mu, \lambda}-v_{\mu}=\max \left(0, \lambda-v_{\mu}\right) .
\end{aligned}
$$

Then, $\left(u_{\mu}, v_{\mu}\right)$ being minimizer, the inequality

$$
\mathcal{F}_{\mu}\left(u_{\mu}, v_{\mu}\right)-\mathcal{F}_{\mu}\left(u_{\mu}, v_{\mu, \lambda}\right) \leq 0
$$

holds. The last expression can be written as

$$
\begin{gathered}
\int_{A(\lambda)}\left(\left|D v_{\mu}\right|^{2}+\left(\operatorname{div}\left(v_{\mu} x\right)\right)^{2}\right)^{\frac{1}{2}}-n \lambda|A(\lambda)| \\
+\sigma \int_{\partial \Omega}\left(v_{\mu}-v_{\mu, \lambda}\right) \sqrt{1+(x \cdot \nu)^{2}} d H_{n-1} \\
+\int_{A(\lambda)} \kappa\left(v^{\frac{n+2}{n}}-\lambda^{\frac{n+2}{n}}\right)-\mu\left(v^{\frac{n+1}{n}}-\lambda^{\frac{n+1}{n}}\right) d x \leq 0 .
\end{gathered}
$$

Now one can use Lemma $4, \sigma \leq 1-a$ and the estimate

$$
c_{\alpha}\left(\lambda-v_{\mu}\right) \leq \lambda^{\alpha}-v_{\mu}^{\alpha} \leq C_{\alpha}\left(\lambda-v_{\mu}\right) \quad \text { for } \alpha>1, \lambda_{1} \leq \lambda \leq \lambda_{2}
$$

to get the inequality

$$
a \int_{A(\lambda)}\left(\left|D v_{\mu}\right|^{2}+\left(\operatorname{div}\left(v_{\mu} x\right)\right)^{2}\right)^{\frac{1}{2}}+a\left(c_{1} \mu-c_{2}\right) \int_{A(\lambda)} v_{\mu, \lambda}-v_{\mu} d x \leq 2 n \lambda|A(\lambda)|
$$

or, equivalently,

$$
\int\left|D w_{\mu, \lambda}\right|+\left(c_{1} \mu-c_{2}\right) \int_{\Omega} w_{\mu, \lambda} d x \leq 2 n a^{-1} \lambda|A(\lambda)| .
$$

The constants $c_{1}$ and $c_{2}$ do not depend on $\mu$. Multiplying both sides with an $\varepsilon>0$ to be chosen later, this gives

$$
\varepsilon \int\left|D w_{\mu, \lambda}\right|+\varepsilon\left(c_{1} \mu-c_{2}\right) \int_{\Omega} w_{\mu, \lambda} d x \leq 2 n \varepsilon a^{-1} \lambda|A(\lambda)| .
$$

Now Ehrling's lemma in the form

$$
\|u\|_{L^{p}(\Omega)} \leq \varepsilon\|u\|_{B V(\Omega)}+c(\varepsilon)\|u\|_{L^{1}(\Omega)}
$$


(where $1<p<\frac{n}{n-1}$ ) can be applied. There is a $\mu_{0}$ such that for $\mu \geq \mu_{0}$ one has $\varepsilon\left(c_{1} \mu-c_{2}\right)>c(\varepsilon)$. For such $\mu,\left\|w_{\mu, \lambda}\right\|_{p} \leq \varepsilon c_{3} \lambda|A(\lambda)|$. Hölder's inequality leads to

$$
\int_{\Omega} w_{\mu, \lambda} d x \leq c_{3} \varepsilon \lambda|A(\lambda)|^{1+\frac{\tau}{p}} \quad(\tau=p-1) .
$$

Setting

$$
y(\lambda)=\int_{\Omega} w_{\mu, \lambda} d x=\int_{A(\lambda)} \lambda-v_{\mu} d x
$$

and using $y_{-}^{\prime}(\lambda)=|A(\lambda)|$ by Lemma 6 , the last inequality can be written as

$$
\left(c_{3} \varepsilon\right)^{\frac{p}{p+\tau}} y_{-}^{\prime}(\lambda) \geq y(\lambda)^{\frac{p}{p+\tau}} \lambda^{-\frac{p}{p+\tau}} .
$$

Assume $y\left(\lambda_{1}\right)>0$, which implies $y(\lambda)>0$ for all $\lambda \in\left[\lambda_{1}, \lambda_{2}\right]$ by monotonicity of $y$. Then Lemma 7 may be used to show

$$
y\left(\lambda_{1}\right)^{\frac{\tau}{p+\tau}} \leq y\left(\lambda_{2}\right)^{\frac{\tau}{p+\tau}}-\left(c_{3} \varepsilon\right)^{-\frac{p}{p+\tau}}\left(\lambda_{2}^{\frac{\tau}{p+\tau}}-\lambda_{1}^{\frac{\tau}{p+\tau}}\right) .
$$

Independently of $\mu$, one has $y\left(\lambda_{2}\right) \leq \lambda_{2}|\Omega|$. Since $\varepsilon^{-\frac{p}{p+\tau}} \rightarrow \infty$ for $\varepsilon \rightarrow 0$, the last inequality is a contradiction to the assumption $y\left(\lambda_{1}\right)>0$ if one has chosen $\varepsilon$ small enough in (32)

Theorem 8. Let $0<\lambda_{1}<\delta$ be given. Then under the assumptions of Theorem 4 there exists a $\mu_{0}$ such that for $\mu \geq \mu_{0}$

$$
v_{\mu} \leq \lambda_{1} \quad \text { a.e. }
$$

Proof. For the proof being very similar to that of Theorem 7 only the ansatz will be given. Let $\lambda_{1}>\lambda_{0}>0$ and $\lambda_{1} \geq \lambda \geq \lambda_{0}$. Define

$$
\begin{aligned}
A(\lambda) & =\left\{x \in \Omega \mid u_{\mu}(x)>\lambda\right\} \\
u_{\mu, \lambda} & =\min \left\{u_{\mu}, \lambda\right\} \\
w_{\mu, \lambda} & =u_{\mu}-u_{\mu, \lambda}=\max \left\{0, u_{\mu}-\lambda\right\} .
\end{aligned}
$$

Now the ansatz is $\mathcal{F}_{\mu}\left(u_{\mu}, v_{\mu}\right)-\mathcal{F}_{\mu}\left(u_{\mu, \lambda}, v_{\mu}\right) \leq 0$

Theorem 9. Let $\partial \Omega$ be of Lipschitz type and $0<\sigma \leq 1$. Let

$$
|\Omega|<\frac{\sigma}{n} \int_{\partial \Omega} \sqrt{1+(x \cdot \nu)^{2}} d H_{n-1}
$$

and $(u, v)$ be the solution of the problem $\mathcal{F}_{\mu}(u, v) \rightarrow \min$ in $K_{\delta}$. Then $u$ is not identical to zero on $\Omega$.

Proof. Suppose $u \equiv 0$ on $\Omega$. Comparing $u$ to the constant $\varepsilon$ gives

$$
\begin{aligned}
\mathcal{F}_{\mu}(u, v)-\mathcal{F}_{\mu}(\varepsilon, v) & =-n \varepsilon|\Omega|+\kappa \varepsilon^{\frac{n+2}{n}}-\mu \varepsilon^{\frac{n+1}{n}}+\sigma \varepsilon \int_{\partial \Omega} \sqrt{1+(x \cdot \nu)^{2}} d H_{n-1} \\
& =\left(\sigma \int_{\partial \Omega} \sqrt{1+(x \cdot \nu)^{2}} d H_{n-1}-n|\Omega|\right) \varepsilon+o(\varepsilon) .
\end{aligned}
$$

The expression in brackets is greater than zero by (36). For $\varepsilon$ small enough, this gives $\mathcal{F}_{\mu}(u, v)-\mathcal{F}_{\mu}(\varepsilon, v)>0$ in contradiction to the minimizing property of $(u, v)$

Acknowledgements. I thank J. Bemelmans (Aachen) for helpful discussions. 


\section{References}

[1] Anzellotti, G.: The Euler equation for functionals with linear growth. Trans. Amer. Math. Soc. 290 (1985), 483 - 501.

[2] Anzellotti, G.: BV solutions of quasilinear PDEs in divergence form. Comm. Part. Diff. Equ. 12 (1987), $77-122$.

[3] Bemelmans, J. and U. Dierkes: On a singular variational integral with linear growth. Part I: Existence and regularity of minimizers. Arch. Rat. Mech. Anal. 100 (1987), 83 - 103.

[4] Concus, P. and R. Finn: On capillary free surfaces in the absence of gravity. Acta Math. 132 (1974), $177-198$.

[5] Dal Maso, G.: Integral representation on $B V(\Omega)$ of $\Gamma$-limits of variational integrals. Man. Math. 30 (1980), 387 - 416.

[6] Emmer, M.: Esistenza, unicita e regolarita nelle superfici di equilibrio nei capillari. Ann. Univ. Ferrara (n. Ser., Sez. VII) 18 (1973), 79 - 94.

[7] Federer, H.: Geometric Measure Theory (Die Grundlehren der mathematischen Wissenschaften in Einzeldarstellungen: Vol. 153). Berlin - Heidelberg - New York: SpringerVerlag 1969.

[8] Finn, R.: Equilibrium Capillary Surfaces (Die Grundlehren der mathematischen Wissenschaften: Vol. 284). Berlin - Heidelberg - New York: Springer-Verlag 1986.

[9] Gerhardt, C.: Existence and regularity of capillary surfaces. Boll. Unione Mat. Ital. (IV. Ser.) 10 (1974), $317-335$.

[10] Gerhardt, C.: On the capillarity problem with constant volume. Ann. Sc. norm. super. Pisa (Cl. Sci., IV. Ser.) 2 (1975), $303-320$.

[11] Gerhardt, C.: Global regularity of the solutions to the capillarity problem. Ann. Sc. norm. super. Pisa (Cl. Sci., IV. Ser.) 3 (1976), 157 - 175.

[12] Giusti, G.: The equilibrium configuration of liquid drops. J. Reine Angew. Math. 321 (1981), $53-63$.

[13] Schindlmayr, G.: Kapillarität im Kegel und in allgemeineren skalierten Gebieten (Berichte aus der Mathematik/Thesis TH Aachen). Aachen: Shaker Verlag 1999.

[14] Taylor, Jean E.: Boundary regularity for solutions to various capillarity and free boundary problems. Comm. Part. Diff. Equ. 2 (1977), 323 - 357.

Received 10.08.1999; in revised form 20.06.2000 